\title{
A PROOF OF THE INVARIANCE OF CERTAIN CONSTANTS OF
}

\section{ANALYSIS SITUS *}

BY

\section{J. W. ALEXANDER, II}

1. Introduction. Every $n$-dimensional manifold may be partially characterized by means of two sets of topological invariants

and

$$
P_{1}, P_{2}, \cdots P_{n-1} \quad(P) \dagger
$$

$$
R_{1}, R_{2}, \cdots R_{n-1} \quad(R) \ddagger
$$

whose rôles are analogous to that played by connectivity in the theory of surfaces. The numbers $P_{i}$ and $R_{i}$ are each greater by unity than thể maximum number of $i$-dimensional cycles (cf. $\$ 4$ below) which may be traced in the manifold, but in calculating the numbers $P_{i}$, certain conventions about sense are taken into account and the attention is confined to the sensed cycles.

Now if a manifold be subdivided into a complex, or generalized polyhedron, then one plus the maximum number of independent $i$-dimensional cycles of the polyhedron (i. e., cycles made up of cells of the polyhedron) is also equal to $R_{i}$, or to $P_{i}$ if the conventions about sense be adopted. This theorem, which is of considerable use in calculating the values of the numbers $(P)$ and $(R)$, has been proved by Poincare on the assumption that the manifold, all the cycles of the manifold, and all the cells of the complex may be regarded as made up of a finite number of analytic pieces. But such an assumption opens the way to a theoretical objection in that the numbers $(P)$ and $(R)$ when calculated from the analytic cycles alone might conceivably fail to be topological invariants. To remove this objection, it would have to be shown that there never could exist a point-for-point continuous reciprocal correspondence between two manifolds possessing different numbers $(P)$ and $(R)$, even if the correspondence were not required to be analytic.

In the following discussion, we shall take into account not only non-analytic cycles but also cycles possessing singularities of however complicated a nature.

* Presented to the Society, September 8, 1913.

† Poincaré, Journal de l'École Polyte chnique, vol. 2 (1895), p. 19; and Palermo Rendiconti, vol. 13 (1899), p. 285.

$\ddagger$ Veblen and Alexander, A n n ls of $\mathrm{M}$ a the matics, vol. 14 (1913), p. 168. 
The inclusion of the latter makes it possible to prove with ease the fundamental theorem about the numbers $(P)$ and $(R)$ as well as a similar theorem about the coefficients of torsion. ${ }^{*}$ The discussion will very largely be limited to 3 dimensional manifolds, since the generalization affords no new difficulties and suggests itself at once.

The author wishes to express his appreciation and thanks to Professor O. Veblen for many valuable suggestions.

2. Model of a 3-Dimensional Manifold. From a topological point of view, a simple closed surface may always be represented in a space of sufficiently many dimensions by a model consisting of a finite number of triangular regions. These regions will abut upon one another in such a way that a side of one always coincides with a side of one of the others. Similarly, a 3dimensional manifold may be represented by a finite number of tetrahedral regions, and so on. $\dagger$ As we are only concerned with manifolds without singularities, the regions which cluster about a common vertex may be supposed to constitute a simply connected portion of the manifold, that is, one which can be mapped upon the interior of a tetrahedron. We can then subdivide the manifold into smaller tetrahedral regions so as to assure ourselves that each region together with all the neighboring regions is contained within a simply connected portion. The model thus finally obtained will be used in place of the manifold itself in all of the discussion which follows.

The vertices, edges, and triangular faces which bound the tetrahedral regions subdivide the manifold into a complex $\pi$, or generalized polyhedron, of three dimensions. They constitute the cells of the polyhedron of dimensionalities 0,1 , and 2 respectively, while the interiors of the tetrahedral regions themselves constitute the cells of dimensionality 3 . The polyhedron $\pi$ is of a very restricted type; it can be assumed that all its edges are of equal length, for if its vertices are $V$ in number, they may all be spaced at equal distances from one another in a space of $V-1$ dimensions.

3. Invariants of a Polyhedron and of a Manifold. If a manifold be subdivided into a polyhedron $\kappa$, whether or not of the restricted type of $\pi$, it is a simple matter to calculate the greatest number of 1-dimensional complexes $c_{1}, c_{2}, \cdots c_{k}$ (closed curves) made up of cells of the polyhedron and such that there is no open 2-dimensional complex also made up of cells of the polyhedron and having for complete boundary one or more of the complexes $c_{1}, c_{2}, \cdots c_{k}$. Taking into account the conventions on sense, this calculation leads to an invariant $P_{1}^{\kappa}-1$, otherwise to an invariant $R_{1}^{\kappa}-1$. Similarly, the greatest number of independent closed 2-dimensional complexes is either $P_{2}^{\kappa}-1$ or $R_{2}^{\kappa}-1$. The problem will be to identify these four invari-

* Poincaré, Proceedings of the London Mathematical Society, vol. 32 (1900), p. 301.

$\dagger$ Veblen and Alexander, loc. cit., § 17. 
ants with the corresponding invariants of the manifold itself, that is with $P_{1}-1, R_{1}-1, P_{2}-1$, and $R_{2}-1$.

4. By a 1- or 2-dimensional cycle $c$ of the manifold will be meant a set of points of the manifold which may be regarded as the image of a closed complex $\lambda$ of one or two dimensions as the case may be. There must be a continuous correspondence between the points of the complex and those of the cycle such that to every point of the complex there corresponds one and only one point of the cycle, but the correspondence need not be one-to-one in the inverse direction. Thus, the cycle may present singularities of any degree of complexity. Whenever two or more points of the complex $\lambda$ correspond to the same point of the cycle $c$, we shall say that they correspond in a one-to-one manner to as many coincident points of $c$. With this convention, the correspondence between $c$ and $\lambda$ becomes one-to-one in both directions and the internal structure of $c$ becomes the same as that of $\lambda$. If any length $\epsilon$ be preassigned, it may be assumed that the cells of the cycle $c$ (the images of the cells of the complex $\lambda$ ) are so small that no two points of the same cell are at a distance of $\epsilon$ or more apart. For if we subdivide the cells of the complex $\lambda$ into sufficiently small cells, the images of the latter will surely have the required property, owing to the uniform continuity of the correspondence between $\lambda$ and $c$.

Before defining what is meant by an independent set of cycles, let us recall that the boundary of an open $i+1$ dimensional complex $\kappa$ consists of one or more closed $i$-dimensional complexes $\lambda_{1}, \lambda_{2}, \cdots \lambda_{i}$ any two of which may have in common one or more cells of dimensionality less than $i$. If such a complex be mapped along with its boundary upon the manifold, the complexes $\lambda_{1}, \lambda_{2}, \cdots \lambda_{i}$ will form a set of cycles $c_{1}, c_{2}, \cdots c_{i}$, two or more of which may coincide. When we disregard the conventions about sense, the cycle $c_{1}$ is said to be dependent upon the cycles $c_{2}, \cdots c_{i}$ provided it occurs an odd number of times in the set, but when the conventions are made, it is deperiuent provided the difference between the number of times it appears in one sense and the number of times it appears in the other is not zero. A set of cycles is said to be independent when no cycle of the set is dependent upon other cycles of the set. A necessary but not sufficient condition for independence is that each cycle of the set be non-bounding, that is that no cycle be the boundary of the image of a complex $\kappa$.

$R_{1}-1$ and $R_{2}-1$ are respectively the greatest number of independent 1- and 2-dimensions cycles which may be traced in the manifold; $P_{1}-1$ and $P_{2}-1$ are the corresponding numbers when conventions on sense are made.

5. Lemma. No cycle lying in a simply connected region can be non-bounding. This may be seen at once if the simply connected region be regarded as the inte rior of a tetrahedron, for if we then join each point of the cycle by a linear 
segment to some point $P$ of the region, the points of the segments will constitute the image of an open complex bounded by the cycle in question. The complex will have as cells a vertex corresponding to the point $P$, an arc corresponding to each line joining $P$ to an arc of the cycle, a triangular region corresponding to each set of lines joining $P$ to an arc of the cycle, and, if the cycle be 2-dimensional, a tetrahedral region corresponding to each set of lines joining $P$ to a triangular region of the cycle.

6. The Fundamental Theorem for Polyhedra of the Restricted Type. Let us first suppose that the manifold be subdivided into a polyhedron $\pi$ of the type discussed in $\S 2$. We shall first prove that every cycle of the manifold is dependent upon one or more cycles of $\pi$.

As was shown in $\S 4$, the cells of the cycle $c$ may be supposed to lie in regions as small as we please; hence, in particular, they may be supposed to lie in such small regions that if every point on the interior and boundary of any one of the cells be joined to the nearest vertex of the polyhedron, or to one of the nearest if there are more than one, these points will all be joined to the same or to adjacent vertices. Now if $c$ be a one-dimensional cycle, the ends $A_{1}$ and $A_{2}$ of each one-cell will be joined respectively to two vertices $B_{1}$ and $B_{2}$ of the polyhedron $\pi$ which either coincide or bound an edge $B_{1} B_{2}$ of $\pi$. At all events, the circuit $A_{1} A_{2} B_{2} B_{1} A_{1}$ will bound the image $s$ of some open complex since it lies in a simply connected region $(\$ 5)$. The totality of complexes $s$ corresponding to the various circuits $A_{1} A_{2} B_{2} B_{1} A_{1}$ constitutes the image $s$ of a complex bounded by the cycle $c$ and, in general, one or more cycles of the polyhedron $\pi$ composed of the $\operatorname{arcs} B_{1} B_{2}$. It may happen that the circuit $c$ constitutes by itself the complete boundary of $S$, as for instance in the case where the vertices $B_{1}$ and $B_{2}$ all coincide. In any case, the cycle $c$ is dependent upon the cycles of the polyhedron $\pi$.

When $c$ is a 2-dimensional cycle, it may be regarded as composed of the images of triangular regions $(\S 2)$. Each 2-cell will have three vertices $A_{1}$, $A_{2}$, and $A_{3}$ which will be joined to three vertices $B_{1}, B_{2}$, and $B_{3}$ of the polyhedron $\pi$ every two of which must either coincide or bound an arc of $\pi$. Now, by the lemma, the cycles $A_{1} A_{2} B_{2} B_{1} A_{1}, A_{2} A_{3} B_{3} B_{2} A_{2}$, and $A_{3} A_{1} B_{1} B_{3} A_{3}$ bound three surfaces $s_{12}, s_{23}$, and $s_{34}$ respectively all of which are situated within the same simply connected portion of the manifold. Moreover, the surfaces $s_{12}, s_{23}$, and $s_{31}$, together with the cells $A_{1} A_{2} A_{3}$ and $B_{1} B_{2} B_{3}$ (the latter being of less than two dimensions if two or more of the vertices $B_{1}, B_{2}$, and $B_{3}$ coincide) form a closed complex which bounds the image $r$ of a 3-dimensional complex, again by the lemma. But the totality of regions $r$ determines a region $R$ which is bounded by the cycle $c$ and, in general, one or more cycles $c^{\prime}$ of the polyhedron $\pi$ made up of cells $B_{1} B_{2} B_{3} .^{*}$

* The term region is here used in a very broad sense to denote the image, with or without singularities, of a 3-dimensional complex. 
Since every cycle of the manifold is dependent upon one or more cycles of the polyhedron $\pi$, we have the relations

$$
R_{1}^{\pi} \geqq R_{1} \quad \text { and } \quad R_{1}^{\pi} \geqq R_{1} .
$$

Moreover, we also have

$$
P_{2}^{\pi} \geqq P_{1} \quad \text { and } \quad P_{2}^{\pi} \geqq P_{2},
$$

since it is readily seen that the above argument holds equally well when the conventions about sense are introduced.

On the other hand, if a set of cycles $a$ made up of cells of the polyhedron fails to be independent with respect to the manifold, so also does it fail to be independent with respect to the complex. For if it is not independent with respect to the manifold, there exists an open complex $\lambda$ which may be so mapped upon the manifold that the boundary of its image $c$ coincides with one or more of the cycles $a$. Let us first suppose that the cycles $a$ are linear so that the complex $\lambda$ is 2-dimensional. Then, supposing as we may that the cells are sufficiently small and the images of triangles, we may find a cell $B_{1} B_{2} B_{3}$ of the polyhedron $\pi$ corresponding to every cell $A_{1} A_{2} A_{3}$ of $c$, by the process indicated above. The cells $B_{1} B_{2} B_{3}$, together with the cells of lower dimensionalities on their boundaries constitute one or more complexes $c^{\prime}$ of $\pi$ bounded by cycles of the set $a$, therefore the cycles are not independent with respect to the polyhedron. It might be objected that the complex or complexes $c^{\prime}$ could fail to exist, as for instance if the vertices $B_{1}, B_{2}$, and $B_{3}$ always coincided. This can never be the case, however, for $c$ and $c^{\prime}$ constitute the boundary of a certain open 3-dimensional region $R$, by the argument made above. But the boundary of $R$ must be a closed complex, whereas $c$ by itself is open. Therefore $c^{\prime}$ must not only exist but must have the same boundary as $c$ in order that $c$ and $c^{\prime}$ together shall constitute a closed complex.

When $a$ is a set of 2-dimensional cycles, $c$ is the image of a complex of three dimensions. We may therefore regard its cells as the images of tetrahedra, besides which we may suppose them as small as we please. If we join the vertices $A_{1} A_{2} A_{3}$ and $A_{4}$ of each cell to the four nearest vertices $B_{1} B_{2} B_{3}$ and $B_{4}$ of $\pi$ respectively, the cells $B_{1} B_{2} B_{3} B_{4}$ will determine the complex $c^{\prime}$. The details of the argument in this and in the previous case are similar. Thus, we also have

$$
R_{1}^{\pi} \leqq R_{1}, \quad R_{2}^{\pi} \leqq R_{2}, \quad P_{1}^{\pi} \leqq P_{1}, \quad P_{2}^{\pi} \leqq P_{2},
$$

and therefore,

$$
R_{1}^{\pi}=R_{1}, \quad R_{2}^{\pi}=R_{2}, \quad P_{1}^{\pi}=P_{1}, \quad P_{2}^{\pi}=P_{2},
$$

which establishes the theorem for the case where $\pi$ is a polyhedron of the restricted type. 
Evidently, all that has been said up to this point may be generalized at once to manifolds of more than three dimensions. The polyhedron $\pi$ will then consist of simplexes, or generalized tetrahedra, together with the simplexes of lower dimensionalities on their boundaries.

7. Corollary. If a manifold be topologically equivalent to the boundary of an $n$-dimensional simplex, or generalized tetrahedron, its invariants $(P)$ and $(R)$ are all equal to unity.

For the cells on the boundary of the simplex determine a polyhedron of the restricted type $\pi$. Moreover, if we remove from one of the $n$-cells of the polyhedron a small $n$-dimensional simplex, there will be left a simply connected region containing all the cycles of the polyhedron. But, by the theorem, these cycles are the only ones which need be considered and, by the lemma, they all bound.

8. The Fundamental Theorem for a General Polyhedron. A polyhedron of the most general type may be transformed into one of the restricted type $\pi$ by means of a series of regular subdivisions. * It merely remains to be shown, therefore, that a regular subdivision does not alter the number of independent cycles of the complex. Let $\kappa^{\prime}$ be the complex before the subdivision and let $\kappa$ be the complex after the subdivision of a cell $E$ of $\kappa^{\prime}$. Then every $i$-dimensional cycle $c_{i}$ of $\kappa$ which was not a cycle of $\kappa^{\prime}$ previous to the subdivision must necessarily pass through the new vertex $V$ of $\kappa$. Now the bases of the (pyramidal) cells of $c_{i}$ which have for common apex the point $V$ constitute one or more cycles $d_{i-1}$ of dimensionality $i-1$, except for the case $i=1$ when they are a set of points, even in number. In either case, since the boundary of a cell is in point-for-point correspondence with the boundary of a simplex, the cycles or points $d_{i-1}$ are the complete boundary of an open complex $d_{i}$ made up of cells on the boundary of $E(\$ 7)$. But the complex $d_{i}$ together with the cells of $c_{i}$ which abut upon $V$ constitutes a closed complex $e_{i}$ which may be shown to bound by exactly the same argument as was used in proving the lemma. If we add $\dagger$ the complex $e_{i}$ to the complex $c_{i}$, the latter is transformed into one which no longer passes through the vertex $V$ and which therefore belonged to $\kappa^{\prime}$ previous to the subdivision. $\ddagger$ Hence,

$$
P_{i}^{\kappa} \leqq P_{i}^{\kappa^{\prime}} \quad \text { and } \quad R_{i}^{\kappa} \leqq R_{i}^{\kappa^{\prime}} .
$$

Moreover, every cycle of $\kappa^{\prime}$ which bounds in $\kappa$ also bounds in $\kappa^{\prime}$. For either the bounded complex belonged to $\kappa^{\prime}$ or else it now passes through the

* A regular subdivision is made by partitioning one of the cells $E$ of the complex into a set of "pyramidal" cells whose bases are the cells on the boundary of $E$ and whose apexes coincide at a point $V$ of $E$. For details, see Veblen and Alexander, loc. cit.

$\dagger$ Veblen and Alexander, loc. cit., p. 169.

$\ddagger$ When the dimensionality of the cycle $c_{i}$ is equal to or greater than that of the cell $E$, the cycle $c_{i}$ includes all the sub-cells of $E$, therefore it is the same cycle as the cycle of $\kappa^{\prime}$ obtained by replacing the sub-cells of $E$ by $E$ itself. 
vertex $V$. In the latter case, it can be replaced by one which does not pass through $V$ by an argument like the one just made. Therefore,

$$
P_{i}^{\kappa}=P_{i}^{\kappa^{\prime}} \quad \text { and } \quad R_{i}^{\kappa}=R_{i}^{\kappa^{\prime}} .
$$

9. When the conventions on sense are introduced, it sometimes happens that a cycle is non-bounding when counted only once, though bounding when counted a sufficient number of times. From a consideration of such cycles are derived the coefficients of torsion of the manifold. An argument like the one made in this paper shows that a cycle having the above property may be replaced first by one of the same kind belonging to a polyhedron of type $\pi$, then by one of the same kind belonging to any polyhedron into which the manifold may be subdivided. The invariance of the coefficients of torsion therefore also follows at once.

Princeton, N. J., September, 1913. 